\title{
BMJ Open Total laparoscopic pancreaticoduodenectomy versus open pancreaticoduodenectomy (TJDBPS01): study protocol for a multicentre, randomised controlled clinical trial
}

\author{
Hang Zhang, ${ }^{1}$ Yechen Feng, ${ }^{1}$ Junfang Zhao, ${ }^{1}$ Rufu Chen, ${ }^{2,3}$ Xuemin Chen, ${ }^{4}$ \\ Xinmin Yin, ${ }^{5}$ Wei Cheng, ${ }^{5}$ Dewei Li, ${ }^{6}$ Jingdong Li, ${ }^{7}$ Xiaobing Huang, ${ }^{8}$ Jing Li, ${ }^{8}$ \\ Jianhua Liu, ${ }^{9}$ Jun Liu, ${ }^{10}$ Yahui Liu, ${ }^{11}$ Zhijian Tan, ${ }^{12}$ Wenxing Zhao, ${ }^{13}$ \\ Heguang Huang, ${ }^{14}$ Deyu Li, ${ }^{15}$ Yahong Yu, ${ }^{1}$ Min Wang, ${ }^{1}$ Renyi Qin (D) ${ }^{1}$
}

To cite: Zhang $\mathrm{H}$, Feng $\mathrm{Y}$, Zhao J, et al. Total laparoscopic pancreaticoduodenectomy versus open

pancreaticoduodenectomy (TJDBPS01): study protocol for a multicentre, randomised controlled clinical trial. BMJ Open 2020;10:e033490. doi:10.1136/ bmjopen-2019-033490

- Prepublication history and additional material for this paper are available online. To view these files, please visit the journal online (http://dx.doi. org/10.1136/bmjopen-2019033490).

$\mathrm{HZ}$, YF and JZ are joint first authors.

Received 09 August 2019 Revised 24 November 2019 Accepted 03 January 2020

Check for updates

(C) Author(s) (or their employer(s)) 2020. Re-use permitted under CC BY-NC. No commercial re-use. See rights and permissions. Published by BMJ.

For numbered affiliations see end of article.

Correspondence to

Renyi Qin;

ryqin@tjh.tjmu.edu.cn

\section{ABSTRACT}

Introduction Pancreatoduodenectomy (PD) is one of the most complex abdominal operations to perform, and it is usually conducted for tumours of the periampullary region and chronic pancreatitis. Minimally invasive surgery has been progressively being developed for pancreatic surgery, first with the advent of hybridlaparoscopy and recently with total laparoscopic surgery. Issues including the safety and efficacy of total laparoscopic pancreaticoduodenectomy (TLPD) and open pancreaticoduodenectomy (OPD) are currently being debated. Studies comparing these two surgical techniques are emerging, and large randomised controlled trials (RCTs) are lacking but are clearly required.

Methods and analysis TJDBPS01 is a multicentre, prospective, randomised controlled, parallel-group, superiority trial in 14 centres with pancreatic surgery experts who have performed $\geq 104$ TLPDs and OPDs. A total of 656 patients who will undergo PD are randomly allocated to the TLPD group or OPD group in a 1:1 ratio. The trial hypothesis is that TLPD has superior or equivalent safety and advantages in postoperative recovery compared with OPD. The primary outcome is the postoperative length of stay.

Ethics and dissemination The Instituitional Review Board Approval of Tongji Hospital, Tongji Medical College, Huazhong University of Science and Technology has approved this trial and will be routinely monitoring the trial at frequent intervals, as will an independent thirdparty organisation. Any results from this trial (publications, conference presentations) will be published in peerreviewed journals and conference proceedings. Trial registration number NCT03138213

\section{INTRODUCTION}

Pancreaticoduodenectomy (PD) is one of the most complex abdominal operations to perform, involving removal of the head of the pancreas, the duodenum and the common bile duct, and it is usually performed for the
Strengths and limitations of this study

- The study is designed as a prospective, randomisedcontrolled trial to achieve conclusion on the highest evidence level to provide the evidence concerning the possible benefits of total laparoscopic pancreaticoduodenectomy (TLPD) over open pancreaticoduodenectomy (OPD) in China, it is (i) multicentric and (ii) internationally registered.

- Only high volume, expert centres are qualified to participate the study. They have to provide (i) laparoscopically trained surgeons with $\geq 104$ TLPD procedures performed, (ii) $\geq 104$ OPD procedures performed and (iii) completed the Tongji Hospital TLPD training programme.

- This is the first randomised trial providing with a 2year survival data after pancreaticoduodenectomy.

- The main limitation is as this study is carried out by a large team of physicians that consists of surgeons, gastroenterologists, radiologists, pathologists and oncologists in several districts, the coordination of the team becomes a big challenge.

- This trial is designed to compare two surgery techniques with an open label so that the patients and surgeons are not blinded for their interventions; however, the data collectors and the analysts are blinded.

treatment of pancreatic cancer and the relief pain in chronic pancreatitis. ${ }^{12}$ Introduced by Gagner and Pomp, who performed the first total laparoscopic PD (TLPD) procedures in 1994, the use of laparoscopy in pancreas surgery has continued to grow rapidly because of its potential technical advantages, such as the precision of movements achieved using laparoscopic instruments and a threedimensional view provided by the unique vision system. ${ }^{3}$ To date, many studies have 
confirmed the advantages of laparoscopic techniques in gastric, renal and cervical surgeries. ${ }^{4-6}$ However, only a few low-quality studies have reported the application of total laparoscopy in pancreas surgery or in PD surgery. Notably, recently performed meta-analyses comparing LPD and open PD (OPD) have been unsatisfactory due to the small number of studies of good quality. ${ }^{7-10}$ Most studies concluded that TLPD was associated with a reduction in estimated blood loss (EBL), a lower transfusion rate and a shorter length of stay (LOS) compared with OPD surgery. In addition, TLPDs have a much longer operative time than OPDs. Finally, there were no significant differences between the two procedures in terms of major complications or mortality. Similarly, all of the studies that reported results on this topic emphasised the need for large randomised controlled trials (RCT). However, RCTs are difficult to perform and are costly. There should be further consideration regarding the need for detection of numerous surgical, clinical and oncological variables. Thus, it is imperative for such a study to have enough patients enrolled. A study recruiting an adequate number of patients is desirable. In year 2017, Palanivelu et al published the first RCT worldwide and concluded that LPD offered a shorter hospital stay compared with OPD. ${ }^{11}$ Poves et al reached the same conclusion the following year. ${ }^{12}$ Both of their studies were conducted in a single-centre setting and recruited only 60 and 66 patients, respectively. The researchers claimed that a multicentre trial will be necessary. LEOPARD-2, the first multicentre RCT, recruited 99 patients but did not reveal the superiority of the TLPD and ended with an estimated mortality rate of $6 \%$; five patients died in the laparoscopic group and one patient died in the open group. ${ }^{13}$ This result limited the application of TLPD and hindered the development of the technique throughout the Netherlands. Therefore, a multicentre-based RCT with available expertise in laparoscopy guaranteeing sufficient enrolment is necessary.

Therefore, in this trial, TJDBPS01, a large registry will be created by collecting data from the 14 qualified centres in China to create a working basis for analysing outcomes of interest and obtaining evidence for comparing TLPD and OPD. Additionally, it will be possible for TJDBPS01 to acquire long-term outcomes of PD patients who have not been reported in any RCT studies.

\section{Objectives}

The broad goal of this trial is to evaluate the safety and efficacy of TLPD and OPD procedures. The main hypothesis is that TLPD has superiority advantages in postoperative recovery and is equivalent in safety to OPD. The primary outcome variable is the postoperative LOS. The secondary outcome variables include EBL, operation time, complication rate, mortality, comprehensive complication index (CCI) and overall survival. ${ }^{14}$

\section{METHODS AND ANALYSIS}

\section{Design}

The TJDBPS01 trial is designed as a multicentre, randomised, controlled, superiority trial with two parallel groups in 14 pancreatic centres among 11 provinces in China, providing a minimum of $>50$ PDs annually (online supplementary file 1). All the participating 14 centres with pancreatic surgery expertise have performed $\geq 104$ TLPDs and OPDs, respectively. ${ }^{15}$ Screening and identification of eligible patients will take place within the participating centres' pancreatic multidisciplinary team (MDT). All patients about to undergo PD will go through a standard evaluation: contrast-enhanced multithin-slice CT scans $(1 \mathrm{~mm})$ with or without endoluminal ultrasound, which will be discussed by the MDT. MRI with diffusionweighted imaging is applied to rule out the suspected metastasis before surgery. Patients recommended for resection on the basis of a high likelihood of a malignant lesion will be contacted and provided with a detailed consensus. Consent (version date 20 March 2018, V.1.0) will be obtained by an investigator who should comply with applicable regulatory requirements and adhere to the ethical principles in the Declaration of Helsinki. It is not necessary for patients to have a histological diagnosis of malignancy before surgery provided that the MDT has confirmed that the lesion is concerning enough to require resection. ${ }^{16}$ Randomisation will be performed in a 1:1 allocation. The patients will be randomised into two groups: ${ }^{1}$ patients who will undergo TLPD and ${ }^{2}$ patients who will undergo OPD. This study is structured after the Standard Protocol Item Recommendations for Interventional Trials (SPIRIT) 2013 guideline defining standard protocol items for clinical trials (figure 1).

\section{Study population}

All adult patients with an indication for PD, including but not limited to pancreatic cancer, bile duct cancer, duodenal cancer and benign diseases such as massforming pancreatitis, will be evaluated and informed of the possibility to take part in the TJDBPS01 trial. After the consent form is signed, participants will be randomised into the two previously mentioned groups.

\section{Patients and public involvement}

Neither patients nor the public are involved in the conception, design or conduct of the study.

\section{Participants eligibility}

Inclusion criteria

Inclusion criteria include ${ }^{1}$ age between 18 and 75 years; ${ }^{2}$ histologically proven periampullary cancer, including pancreatic cancer, bile duct cancer and duodenal cancer; ${ }^{3}$ cStage I pancreatic cancer; ${ }^{4}$ an indication for elective PD surgery, ${ }^{5}$ feasibility for both TLPD and OPD (the subject can also undergo surgery according to the MDT) $;{ }^{6}$ patients understand the nature of this trial and are willing to comply; ${ }^{7}$ patients are able to provide written 


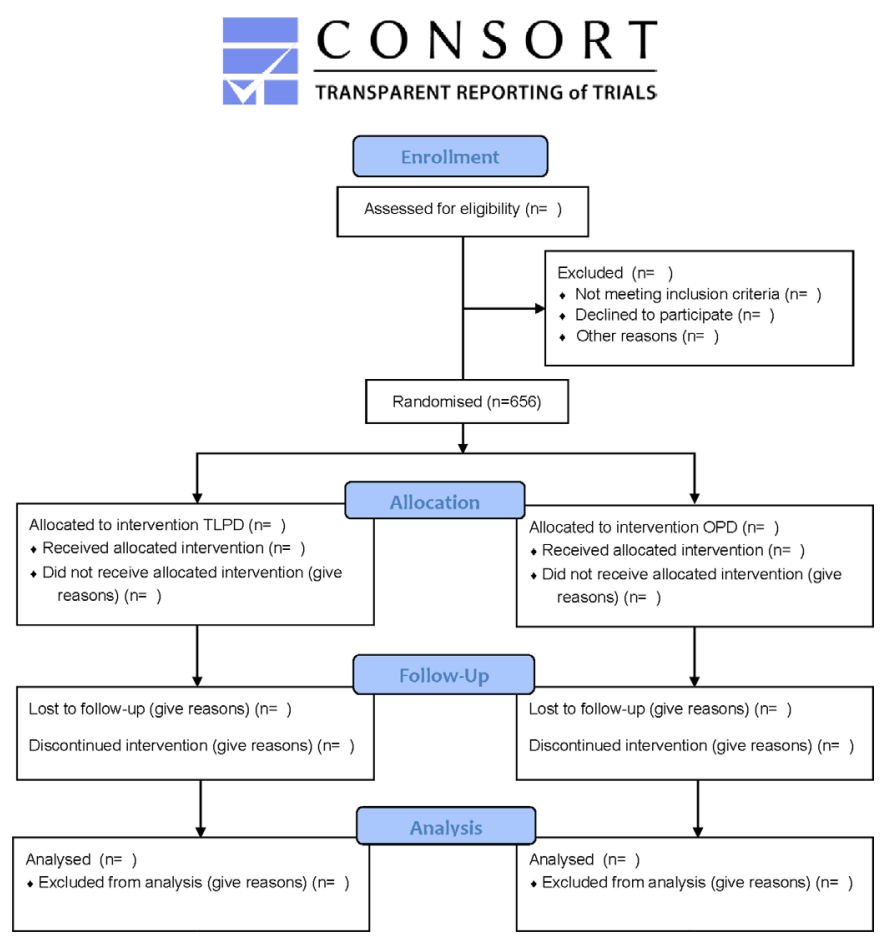

Figure 1 Flow diagram for TJDBPS01. CONSORT, Consolidated Standards of Reporting Trials; OPD, open pancreaticoduodenectomy; TLPD, total laparoscopic pancreaticoduodenectomy.

informed consent and $^{8}$ patients are treated with curative intent in accordance with international guidelines. ${ }^{17}$

\section{Exclusion criteria}

Exclusion factors include ${ }^{1}$ distant metastases including peritoneal carcinomatosis, liver metastases, distant lymph node metastases and involvement of other organs; ${ }^{2}$ patients may undergo left, central or total pancreatectomy or palliative surgery other than $\mathrm{PD} ;{ }^{3}$ patients with high operative risk, as defined by the American Society of Anesthesiologists (ASA), with a score $\geq 4 ;{ }^{4}$ synchronous malignancy in other organs or second cancer requiring resection during the same procedure $;{ }^{5}$ pregnancy; or ${ }^{6}$ patients underwent or needed neo-adjuvant therapy including medication or radiotherapy.

\section{Interventions}

\section{TLPD surgical procedures}

The standard operative technique is described as follows (figure 2). Any small variations according to the surgeon's preference, such as a different order for surgical steps, a variation in approach or pancreatic anastomosis technique, will be allowed but must be recorded authentically in the case record form.

All procedures are to be performed by two regularly trained pancreatic surgeons. Patients will be under general anaesthesia and placed in a supine position; an anti-Trendelenburg position $\left(10^{\circ}-30^{\circ}\right)$ may be used, if necessary. The right arm is placed along the body and the left arm in $90^{\circ}$ abduction for accessing arterial monitoring. A 12-mm trocar (Versaport, COVIDIEN) is
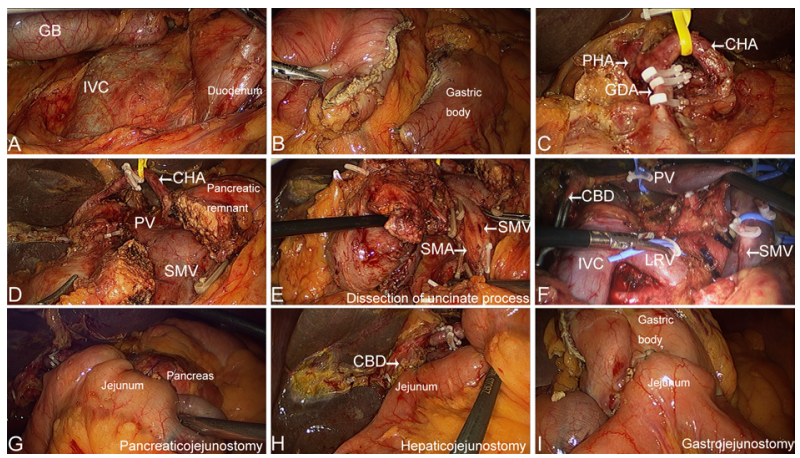

Figure 2 Representative photographs and laparoscopic views of TLPD. (A) Kocher manoeuvre; (B) division of the gastric body; (C) dissection of the GDA; (D) division of the pancreatic neck; $(E)$ dissection of the uncinate process; $(F)$ picture after specimen removal; (G) pancreaticojejunostomy; $(\mathrm{H})$ hepaticojejunostomy and (I) gastrojejunostomy. CBD, common bile duct; CHA, common hepatic artery; GB, gall bladder; GDA, gastroduodenal artery; IVC, inferior vena cava; LRV, left renal vein; PHA, proper hepatic artery; PV, portal vein; SMA, superior mesenteric artery; SMV, superior mesenteric vein. TLPD, total laparoscopic pancreaticoduodenectomy.

placed slightly lower than the umbilicus, and pneumoperitoneum is established. Two $12 \mathrm{~mm}$ trocars are then placed, both lateral to the first trocar on the right and left in the midclavicular lines. Another two $5 \mathrm{~mm}$ trocars are placed at the right and left infracostal arch on both sides of the anterior axillary line (3-4 cm subcostal). Diagnostic laparoscopy is performed to rule out any abnormalities and metastasis. The cystic duct and artery are transected, and the gallbladder is moved and set aside. The round ligament is retracted to the anterior abdominal wall, possibly with either a suture, according to the surgeons' experience. The lesser sac is opened and a Kocher manoeuvre is performed, and the duodenum is therefore mobilised. The gastro-epiploic artery and vein are transected. The distal stomach is transected on the left side of the pylorus with an endostapler (COVIDIEN, Endo GIA, Auto Suture) after removal of the nasogastric tube. The common hepatic arteries are identified on the inferior border of the pancreas and suspended with a rubber band. Lymph node station $8 \mathrm{a}$ is dissected. Subsequently, the right gastric artery and gastroduodenal artery are ligated and transected with at least two Hemo-lok clips (WECK) and one firm ligation (Ethicon). The portal vein (PV) is then identified at the superior border of the pancreatic neck, and the superior mesenteric vein (SMV) is identified at the inferior border of the pancreas. A tunnel is created posterior to the pancreatic neck and anterior to the SMV and PV. The pancreas is slung with a vascular blocking band and then transected with ultrasonic shears (THUNDERBEAT, OLYMPUS), but the pancreatic duct is transected sharply with scissors and intubated to ensure that it is open. The jejunum is transected approximately $15 \mathrm{~cm}$ from the ligament of Treitz with an endostapler (COVIDIEN, Endo GIA, Auto 
Suture). After retracting the duodenum and jejunum to the right side of the mesenteric root, the duodenum is stretched, and the uncinate process is mobilised. The branches of the SMA and SMV are carefully handled until the uncinate process is fully dissected. Retroportal lymph nodes are resected according to the International Study Group on Pancreatic Surgery guidelines. ${ }^{18}$ The common hepatic duct is tunnelled and transected for anastomosis.

Preparation of the pancreatic stump and jejunal loop: the pancreatic stump remnant is dissected to approximately $0.5 \mathrm{~cm}$ (no more than $1.0 \mathrm{~cm}$ ) in length, and careful haemostasis is established with the ultrasonic shears or absorbable sutures. A 6-8 Fr plastic catheter is then inserted as a stent into the pancreatic duct remnant to prevent pancreatic duct stenosis after suture placement. The jejunal limb is brought up to the right of the middle colic vessels in a retrocolic manner, and the blind end is placed near the pancreatic remnant. Negative resection margin status on the frozen section of the specimen will be confirmed before intracorporeal reconstruction if malignancy is suspected.

Pancreaticojejunal (PJ)anastomosis: an imbedding endto-side PJ including four layers of mattress sutures is performed. ${ }^{19}$ The first layer of the anastomosis is created between the posterior wall of the pancreatic stump and the posterior seromuscular layer of the jejunum. Two completely transpancreatic 4-0 prolene (Premilene, BRAUN) mattress sutures are placed at a point approximately $0.2 \mathrm{~cm}$ from the superior and inferior edge of the main pancreatic duct, respectively. The most critical surgical step in placement of the first and second mattress sutures is interlocking them in the posterior wall of the jejunum. Care should be taken to preset the sutures to avoid passing them through or injuring the main pancreatic duct. Both mattress sutures are preplaced approximately $0.5 \mathrm{~cm}$ from the cut edge of the pancreatic remnant. The number of these sutures varies depending on the size of the pancreatic duct but typically ranges between two and four sutures. After a small, full-thickness jejunotomy is created in line with the pancreatic duct, the second layer of the anastomosis is created between the posterior wall of the pancreatic stump and the posterior wall of the small jejunal hole. Two completely transpancreatic 4-0 prolene mattress sutures are placed following the first two sutures, but a full-thickness mattress suture in the posterior wall of the small hole is made in the jejunum approximately $0.2 \mathrm{~cm}$ from the edge. The third and fourth mattress sutures are interlocked in the posterior wall of the jejunal hole. The posterior wall of the jejunal loop is then tightly anastomosed with the posterior wall of the pancreatic stump without any remaining suture intervals. The third layer of the anastomosis is made between the anterior wall of the pancreatic stump and the anterior wall of the jejunum. Two incompletely transpancreatic interlocking 4-0 prolene mattress sutures are placed as described for the second layer of the anastomosis. However, the mattress sutures do not completely penetrate the pancreatic parenchyma but only enter the tissue half-way. The fifth and sixth mattress sutures are supposed to interlock in the anterior wall of the jejunal hole. The fourth layer of the anastomosis has been made between the anterior wall of the pancreatic stump and the anterior wall of the jejunum. Four incompletely transpancreatic interrupting 4-0 prolene mattress sutures are placed to close the gaps in the superoinferior margin of the anastomosis and strengthen the sutures on its anterior wall, achieving a pancreas-enteric anastomosis. Approximately $10 \mathrm{~cm}$ distally, an end-to-side hepaticojejunostomy is performed with running (bile duct dilatation of $>5 \mathrm{~mm}$ ) or interrupted (bile duct dilatation of $\leq 5 \mathrm{~mm}$ ) sutures (Novosyn, BRAUN). Approximately $40 \mathrm{~cm}$ distal to the biliary anastomosis, an antecolic side-to-side gastroenterostomy is performed with the staple technique, and two layers of running 3-0 sutures (Novosyn, BRAUN) are used to close the gastric stump. Two $27 \mathrm{Fr}$ surgical drains are placed through Winslow and the upper region in the PJ stoma. After haemostatic control, the trocars are removed. The specimen is extracted through an uppermiddle incision, which is subsequently closed in layers together with the closure of the trocars. A video clip for illustrative purpose of the basic laparoscopic surgical procedure is available as online supplementary files 23 .

\section{OPD techniques}

The OPD will be performed by the same group of surgeons. A right-sided rectus muscle abdominal incision is preferred for better exposure. The key steps are similar to those taken in the TLPD group. Since outcomes of open pancreatoduodenectomy worldwide are promising and convincing, the surgical technique in the OPD arm reflects their usual practice; also, the anastomoses will be performed according to each centre's protocol. Procedure variations according to the surgeon's preferences are allowed but must be recorded on the case record form. Two surgical drains are placed as in the TLPD procedure.

\section{Conversion from TLPD to OPD}

Conversion is defined as any TLPD group in which a skin incision is used for reasons other than trocar placement or specimen removal. Subjects allocated to TLPD who undergo intraoperative conversion to open pancreatoduodenectomy will be analysed in the TLPD group according to intention-to-treat principles. Reasons for conversion should be carefully recorded in the surgical record.

\section{Postoperative management}

Postoperatively, antibiotics will be administrated as well as either parenteral or enteral nutrition. Somatostatin analogues are not routinely used for preventing postoperative pancreatic fistula. The abdominal drains are kept in place for observation and will be removed if they are not productive and/or the presence of a pancreatic fistula or bile leakage is excluded. Amylase determination of the drains is measured on the third postoperative day (POD) or when a fistula is suspected following the 
International Study Group on Pancreatic Fistula (ISGPF) criteria. Patients are willing to be discharged home when reached criteria as follow: ${ }^{1}$ do not need any intravenous infusion, ${ }^{2}$ can orally take solid or semisolid food, ${ }^{3}$ do not need analgesics or fully comfort by orally analgesics, ${ }^{4}$ incision fully recovered without infection, ${ }^{5}$ could get off bed without assistance, ${ }^{6}$ walking at least $250 \mathrm{~m}$ in plain road, ${ }^{7}$ major organ functioning well and nearly normal haematological parameters. The surgeons operating are not involved in the postoperative management hence cannot influence the discharge of the patients.

\section{Follow-up}

All patients will be followed-up for a period of 2 years.

\section{Withdrawal}

All the patients will be free to participate in this study and can decide to withdraw at any time. If a patient withdraws, his/her information will not be recorded in the study. However, the research team can still collect outcome data from the healthcare records.

\section{Outcome measures}

\section{Primary outcome measure}

The primary outcome variable is the postoperative LOS.

\section{Secondary outcome measures}

The secondary outcome variables include the EBL, operation time, complication rate, mortality, CCI and overall survival.

\section{Missing data}

Bias due to missing data will be investigated by comparing the baseline characteristics of participants with and without missing values. Depending on the extent of missing levels, the predictors of missing values will be identified. The primary outcome analysis will be conducted according to the intention to treat analysis and will be adjusted for the predictors of missing values as the sensitivity analysis. In addition, multiple imputations will be used to impute missing data, and the imputed data will also be analysed as part of the sensitivity analyses.

\section{Participant timeline}

The trial time schedule of enrolment is estimated to be a 2-year period, followed by a 2-year follow-up visit after discharge from the hospital. Once the eligibility of the patients is confirmed, randomisation will be applied. When to apply the interventions is not strictly defined: every collaborating centre can make its own preferred adjustment, but this will usually be within 48 hours after the randomisation. The assessments and visits for patients are mandatory in the first month, at 3 months, 6 months, 12 months and 24 months, with either telephone or in-hospital follow-up (figure 3).

\section{Sample size}

Considering the primary outcome variable is the LOS and according to the latest systemic review, ${ }^{20}$ Miniamlly invasive pancreaticoduodenectomy (MIPD) was associated with a shorter LOS than OPD was (mean difference (MD) -2.95 days, $95 \%$ CI -3.91 to -2.00 days). Accordingly, the sample size is determined based on the primary objective of comparing the LOS between two surgical methods. Based on a MD of 2.95 days in the two surgical groups, using the two-sided test with $80 \%$ power at a significance level of $5 \%$, the minimal sample size needed to detect a significant difference is calculated to be 274 patients in the TLPD group and 274 in the OPD group $(\alpha=0.05, \beta=0.2$, power $=0.8$ ). Considering washout or loss of follow-up, we enlarged the sample size by $20 \%$ to compensate for withdrawal/dropout. Then, the final sample size is 656 . Thus, there are 328 patients in each group.

\begin{tabular}{|c|c|c|c|c|c|c|c|c|c|}
\hline \multirow{2}{*}{ TIME POINT } & \multicolumn{9}{|c|}{ STUDY PERIOD } \\
\hline & $\begin{array}{c}\text { Enrollment } \\
\text { Outpatient } \\
\text { clinic/Admission }\end{array}$ & $\begin{array}{c}\text { Allocation } \\
\text { Before } \\
\text { Surgery (TO) }\end{array}$ & $\begin{array}{l}\text { Treatment } \\
\text { Surgery }\end{array}$ & $\begin{array}{c}\text { Discharge } \\
\text { After } \\
\text { Surgery }\end{array}$ & \multicolumn{4}{|c|}{ Post-allocation } & $\begin{array}{c}\text { Close-out } \\
24 \text { months (T5) }\end{array}$ \\
\hline Eligibility screen & $x$ & & & & & & & & \\
\hline Informed consent & $x$ & & & & & & & & \\
\hline Allocation & & $x$ & & & & & & & \\
\hline OPD & & & $x$ & & & & & & \\
\hline \multicolumn{10}{|l|}{ ASSESSMENTS } \\
\hline Baseline characteristics & $x$ & & & & & & & & \\
\hline Blood routine & $x$ & & & $x$ & $x$ & $x$ & $x$ & $x$ & $x$ \\
\hline Blood biochemistry & $x$ & & & $x$ & $\times$ & $\times$ & $x$ & $\times$ & $\times$ \\
\hline Pathological findings & $x$ & & & $\times$ & & & & & \\
\hline Adjuvant therapy (If necessary) & & & & $x$ & $x$ & $x$ & $x$ & $\times$ & $\times$ \\
\hline
\end{tabular}

Figure 3 Standard Protocol Item Recommendations for Interventional Trials (SPIRIT). OPD, open pancreaticoduodenectomy; TLPD, total laparoscopic pancreaticoduodenectomy. 
The null hypothesis (H0) is that the mean LOS of TLPD group is the same in OPD group. In another word H0: Mean LOSTLPD=Mean LOSOPD. The alternative hypothesis (HA) is that the mean LOS of TLPD group is not the same in OPD group. HA: Mean LOSTLPD $\neq$ Mean LOSOPD. Two sample t-test are used then if the test statistic turns out that $p>0.05$, then it fails to reject the null. The mean LOS of TLPD group is the same in OPD group. Otherwise, if $p<0.05$, the null hypothesis is rejected. The mean LOS of TLPD group is not the same in OPD group. Base on the previous study and the hypothesis, a -2.95 days of MDs should be obtained.

\section{Recruitment}

Each centre will screen eligible patients through the outpatient department or through advertising by the qualified surgeons. The duration of the recruitment period is estimated to be a 24-month interval depending on each centre's recruiting rate. No financial incentives will be provided to trial investigators or patients for enrolment in the recruitment period.

\section{Participating surgeons and centre criteria}

To prevent surgeon bias, participating surgeons must satisfy the following criteria: ${ }^{1}$ they must have experience in performing more than 104 TLPDs, ${ }^{2}$ they must have experience in performing more than 104 OPDs and $^{3}$ they must have completed the Tongji Hospital TLPD training programme.

All the participating centres represent the largest pancreatic surgery centres in their region, ensuring that 50 PDs are performed annually. If willing to join the trial, each surgeon will be mandated to offer two recently unedited surgery videos for evaluation. The video should be sent to the Board and saved appropriately. Only when the Board approves the surgical techniques can the surgeon and his or her centre participate as a collaborator.

\section{Randomisation and allocation}

We applied the stratified randomised block design for the 14 centres with a block number of 4 , and the design is conceived using SAS statistical software V. 9.3. The randomisation is centralised through a computergenerated system and performed in a parallel fashion. A data manager who is independent of the data analysis or patient enrolment generated the randomisation schedule. Allocation will be announced and handled out by the study coordinators only after a baseline assessment and the patient consenting to participate in the study. The randomisation schedule will not be available to study recruiters or physicians. Allocation will be conducted by the independent data manager. Specifically, when appropriate patients are enrolled, the researcher will inform the data manager, and then the random number and exact treatment group will be returned simultaneously.

\section{Blinding}

The patients and surgeons are unblinded while the data collectors, outcome assessors and data analysts are blinded. The primary endpoint of this study is the LOS and is not influenceable by the data collectors, outcome assessors and data analysts. Since they are blinded and also are not involved in the preoperative, perioperative and postoperative management of the patients, thus they have no determination of the LOS. The surgeons only perform the surgery and did not involve in the postoperative management. The patients have little influence concerning the LOS, since once the discharge criteria are reached, they will be discharge immediately. Most of the criteria are objective conditions like do not need any intravenous infusion, incision fully recovered without infection, major organ functioning well and nearly normal haematological parameters. These conditions would not be influenced by the patients even if they are unblinded.

\section{Data collection}

Patient demographics: year of birth, sex, body mass index, surgical risk (ASA score), comorbidities, previous surgical history, main complaint, preoperative biliary drainage, preoperative blood samples (haemoglobin level, white blood cell count and granulocyte: lymphocyte (G:L) ratio), plasma level of total bilirubin, carbohydrate antigen (CA) 19-9, CA 125, carcinoembryonic antigen, alpha-fetoprotein, date of admission and income.

Surgery information: operation date, surgical approach (open or total laparoscopic), type of digestive tract reconstruction, anastomosis approach (intracorporeal, extracorporeal), anastomosis performance (linear stapler, circular stapler, hand-sewn or combinations), placement of intra-abdominal drain, placement of nasogastric tube, total operative time, each anastomosis time (pancreaticojejunostomy, cholangiohepaticojejunostomy, pancreatogastrostomy), texture of pancreas, diameter of the main pancreatic duct, EBL, intraoperative blood transfusion, conversion to open surgery, intraoperative complications, intraoperative death and extent of lymphadenectomy.

Histopathological information: surgical margin status (R0 resection rates), number of retrieved lymph nodes, number of positive lymph nodes, tumour location, size of the tumour, depth of invasion (T classification), lymph node status (N classification), American Joint Committee on Cancer staging (AJCC) ${ }^{21}$ and histological type. ${ }^{22}$

Postoperative clinical findings: length of postoperative hospital stays, postoperative blood transfusion, patient mobilisation (POD), liquid diet (POD), soft solid diet (POD), drain removal (POD), length of intravenous analgesic use, cost for hospitalisation, cost for surgery, drain production and amylase, haemoglobin levels, white cell count, tumour marker level after surgery, type of complication, reoperation and Clavien-Dindo grade. ${ }^{23}$

Follow-up: date of last follow-up visit, adjuvant chemotherapy, adjuvant radiotherapy, patient status at last follow-up visit (alive, dead or lost to follow-up) and disease-free or not during follow-up. All the data will be placed into the local database via a data registry server managed by the data collection group. In this trial, the 
follow-up and statistical staff will be masked to the intervention arms.

\section{Data management}

In this study, the EDC data collection system will be used for data collection. The roles of the staff involved in data collection include the clinical research coordinator, clinical research associate and others.

All staff involved in the data collection process are to be qualified to access the research database. Data collection must be collected in accordance with standard specification processes. Photographers should work in accordance with the visit process requirements, and completed eCRF will be used to enter the original data into the system with a guide; the system cannot automatically identify the data needed to entered through manual input by the data entry person.

\section{General statistical methods}

SAS statistical software (V.9.4), the R programme (R Foundation for Statistical Computing, V. 3.4.4), Power Analysis and Sample Size (PASS, V.11.0 NCSS) and other required statistical software programmes will be used, when appropriate. All statistical tests will be tested with two sides, and $\mathrm{p}<0.05$ is considered to represent a statistically significant difference, with a CI of 95\%. Quantitative data statistics will be described by the number of cases, means, SD, median, quartile (IQR), maximum and minimum (MinMax) values. Qualitative data statistics will be described by the frequency, constituent ratio or percentage. The effect research and the analysis comparison will select the data set according to the specific research goal with the appropriate statistical analysis method. The intergroup comparison of qualitative data will use the $\chi^{2}$ test, and the independent sample $T$ test will be used to compare the quantitative data between the groups.

The intergroup comparison of control covariance can be compared by covariance analysis or the generalised linear model.

We used the SPIRIT checklist when writing this study protocol. $^{24} 25$

\section{Data monitoring}

Efficacy and safety of the duration of data acquisition is 90 days after the patient signs the informed consent (ICF) and the visit. Security data will evaluate the severity of adverse events based on the Common Terminology Criteria for Adverse Events (CTCAE V.4.0) standard. All adverse events will be recorded on the CRF, from the signing of the ICF to the end of the study. LinkDoc acts as the independent third party and will conduct data monitoring throughout the whole trial.

\section{Harms}

All the harms, including adverse events or even severe adverse events, will be recorded in detail according to the CTCAE V.4.0. The data will also be collected by the Hospital's Ethical Committee and in the Chinese Clinical Trial Registry.

\section{Protocol amendments}

Any modifications of the protocol that may impact the conduct of the study, potentially benefit the patients or that may affect patient safety, including changes in study design, sample size and study procedures, will require a formal amendment to the protocol. Additionally, this would need to be submitted to the Hospital Ethics Committee and health authorities must be notified in accordance with local regulations.

\section{Confidentiality}

All study-related information and participant information will be stored securely at the study site with locked cabinets in areas with limited access. All local databases will be secured with a password-protected access system.

\section{Publications}

Each participating investigator, with equal right, will be able to access the data of the registry, perform statistical analysis, discuss the results and freely write scientific manuscripts. The manuscript must be approved by all the authors before publication.

\section{Ethics and dissemination}

All patients signed an informed consent document before entering the study (online supplementary file 4). Consent is obtained by the consultant or designated team member and is preserved by the data collection group. The recruitment will not start at other centres in the trial until local ethical approval has been obtained. Participating in both groups does not imply any additional risk for the subjects included, since the groups will not be deprived of the application of the most up-to-date recommendations. Any results from this trial (publications, conference presentations) will be published in peer-reviewed journals and conference proceedings.

\section{DISCUSSION}

The TJDBPS01 trial is a multicentre, RCT. The trial hypothesis is that TLPD has advantages for postoperative recovery and is equivalent in operation time and oncological results to OPD. The trial is conducted after the completion of a structured training programme for TLPD in China. The length of the learning curve is analysed, considering the argument on timing. Based on the results of a retrospective study of LPD in China, a minimum of 40 total LPDs is decided as the cut-off point. ${ }^{15}$ When the surgeon finished the 104th LPD, the surgical technique would be regarded as mature and stable. Thus, a minimum of 104 TLPDs is decided as the threshold to participate in the TJDBPS01 trial.

As previously mentioned, this is not the only RCT on this topic. Palanivelu et al published their RCT trial results in 2017. ${ }^{11}$ They conducted a trial comparing LPD and OPD for the treatment of periampullary tumours in a single tertiary-care teaching institute in India and concluded that laparoscopy offered a shorter hospital stay than open 
pancreatoduodenectomy in their randomised trial. The trial recruited 64 patients over a 2-year interval period. This study demonstrated a significant advantage for the laparoscopic group in terms of shortened median duration of postoperative hospital stay, which was decreased by half compared with that of open surgery. Additionally, the laparoscopic approach was better regarding blood loss and transfusion requirements in their study, but the difference was less pronounced when procedures with venous resection were excluded. Other outcomes, including the complication rate, lymph node retrieval, and $\mathrm{R} 0$ resection, were comparable in the two groups. The authors also reported that their study had limitations, including a relatively small sample size, which resulted in some outcomes not being assessed, such as recurrence and survival (disease-free and overall) and long-term complications. The same conclusion was supported by the study of Poves $e t$ al. ${ }^{12}$ Unlike the promising conclusions of the previous two studies, the first multicentre trial, LEOPARD-2, demonstrated a concern for the safety of TLPD. ${ }^{13}$ Their trial found a higher mortality rate in the laparoscopic group, and the authors stated that a small volume centre and an early learning curve might have influenced the outcomes, although all enrolled surgeons were required to have completed training programmes for TLPDs. With all these pros and cons, it is necessary and important to carry out an RCT in a multicentre setting based on the premise that both safety and quality are guaranteed.

In conclusion, the TJDBPS01 trial is a multicentre, RCT investigating the safety and effectiveness of TLPD and OPD performed by surgeons who have performed $\geq 104$ TLPDs and OPDs, respectively. This trial aims to evaluate TLPD and OPD in daily practice within high-volume pancreatic centres. When this trial is completed with the hypothesis confirmed, it will popularise the application of TLPD and improve patient outcomes.

\section{Author affiliations}

${ }^{1}$ Department of Biliary-Pancreatic Surgery, Tongji Hospital of Tongji Medical College of Huazhong University of Science and Technology, Wuhan, Hubei, China

${ }^{2}$ Department of General Surgery, Sun Yat-Sen Memorial Hospital, Guangzhou,

Guangdong, China

${ }^{3}$ Department of Hepatobiliary Pancreatic Surgery, Guangdong Provincial People's Hospital, Guangzhou, Guangdong, China

${ }^{4}$ Department of Pancreaticobiliary Surgery, Third Affiliated Hospital of Soochow University, Changzhou, Jiangsu, China

${ }^{5}$ Department of Hepatobiliary Surgery, Hunan Provincial People's Hospital,

Changsha, Hunan, China

${ }^{6}$ Department of Hepatobiliary Surgery, Chongqing Medical University First Affiliated Hospital, Chongqing, Sichuan, China

${ }^{7}$ Department of Pancreatico-Hepatobiliary Surgery, Affiliated Hospital of North Sichuan Medical College, Nanchong, Sichuan, China

${ }^{8}$ Department of Pancreatico-Hepatobiliary Surgery, The Second Affiliated Hospital, Army Medical University, Chongqing, Chongqing, China

${ }^{9}$ Department of Hepato-Pancreato-Biliary Surgery, Second Hospital of Hebei

Medical University, Shijiazhuang, Hebei, China

${ }^{10}$ Department of Hepato-Pancreato-Biliary Surgery, Shandong Provincial Hospital, Jinan, Shandong, China

${ }^{11}$ Department of Hepatobiliary and Pancreatic Surgery, Jilin University First Hospital, Changchun, Jilin, China
${ }^{12}$ Department of Hepatobiliary and Pancreatic Surgery, Guangdong Hospital of Traditional Chinese Medicine, Guangzhou, Guangdong, China

${ }^{13}$ Department of Hepato-Pancreato-Biliary Surgery, Xuzhou Medical College Affiliated Hospital, Xuzhou, Jiangsu, China

${ }^{14}$ Department of Hepato-Pancreato-Biliary Surgery, Fujian Medical University Union Hospital, Xiamen, Fujian, China

${ }^{15}$ Department of Hepatobiliary Pancreatic Surgery, Henan Provincial People's Hospital, Zhengzhou, Henan, China

Acknowledgements We thank Prof. Jingdong Ma and Dr Tingting Qin from the Department of Epidemiology and Biostatistics, School of Public Health, Tongji Medical College, Huazhong University of Science and Technology, for the statistical support. We thank LinkDoc for the data monitoring. We thank AJE for the English language, grammar, punctuation, spelling and overall style editing.

Contributors HZ, YCF and JFZ are joint first authors. RYQ, MW and YHY obtained funding for the study. RYQ, HZ and MW designed the study. RFC, XMC, WC, DWL, JDL, HGH, DYL, JiL, JHL, JuL, YHL, ZJT, WXZ and RYQ performed the operations. $\mathrm{XBH}$ and XMY give supervision for the operation. HZ YCF and JFZ drafted the manuscript. RYQ and MW contributed to critical revision of the manuscript for important intellectual content and approved the final version of the manuscript. All authors have read and approved the final manuscript.

Funding This study is supported by grants from the Hubei Natural Science Foundation (WJ2017Z010), Tongji Hospital Clinical Research Flagship Program 2019 CR203.

Map disclaimer The depiction of boundaries on this map does not imply the expression of any opinion whatsoever on the part of BMJ (or any member of its group) concerning the legal status of any country, territory, jurisdiction or area or of its authorities. This map is provided without any warranty of any kind, either express or implied.

\section{Competing interests None declared.}

Patient consent for publication Not required.

Ethics approval Ethics approval is obtained from the Instituitional Review Board Approval of Tongji Hospital, Tongji Medical College, Huazhong University of Science and Technology (TJ-IRB20180512) in May 2018. This study has gained ethical approval at both the central and local levels of each participating centers, and central ethical approval has been confirmed by the Instituitional Review Board Approval of Tongji Hospital, Tongji Medical College, Huazhong University of Science and Technology.

Provenance and peer review Not commissioned; externally peer reviewed.

Open access This is an open access article distributed in accordance with the Creative Commons Attribution Non Commercial (CC BY-NC 4.0) license, which permits others to distribute, remix, adapt, build upon this work non-commercially, and license their derivative works on different terms, provided the original work is properly cited, appropriate credit is given, any changes made indicated, and the use is non-commercial. See: http://creativecommons.org/licenses/by-nc/4.0/.

ORCID iD

Renyi Qin http://orcid.org/0000-0001-5079-5137

\section{REFERENCES}

1 Siegel RL, Miller KD, Jemal A. Cancer statistics. Ca: a cancer Journal for clinicians. 2016;66:7-30.

2 Tsamalaidze L, Stauffer JA. Pancreaticoduodenectomy: minimizing the learning curve. J Vis Surg 2018;4:64.

3 Gagner M, Pomp A, pancreatoduodenectomy Lpylorus-preserving. Laparoscopic pylorus-preserving pancreatoduodenectomy. Surg Endosc 1994;8:408-10.

4 Agrusa A, di Buono G, Chianetta D, et al. Three-Dimensional (3D) versus two-dimensional (2D) laparoscopic adrenalectomy: a casecontrol study. International Journal of Surgery 2016;28:S114-7.

5 Miyamoto R, Inagawa S, Nagai K, et al. Three-Dimensional reconstruction of vascular arrangement including the hepatic artery and left gastric vein during gastric surgery. Springerplus 2016;5:835.

6 Raspagliesi F, Bogani G, Martinelli F, et al. Incorporating 3D laparoscopy for the management of locally advanced cervical cancer: a comparison with open surgery. Tumori Journal 2016;102:393-7.

7 Chen K, Pan Y, Liu X-long, Liu XL, et al. Minimally invasive pancreaticoduodenectomy for periampullary disease: a 
comprehensive review of literature and meta-analysis of outcomes compared with open surgery. BMC Gastroenterol 2017;17:120.

8 de Rooij T, MZ L, Steen MW, et al. Minimally invasive versus open pancreatoduodenectomy: systematic review and meta-analysis of comparative cohort and registry studies. Annals of surgery 2016;264:257-67.

9 Nickel F and al. laparoscopic versus open pancreaticoduodenectomy: a systematic review and meta-analysis of randomized controlled trials. Ann Surg 2019. Epub ahead of print]..

10 Zhang $\mathrm{H}$, Wu X, Zhu F, et al. Systematic review and metaanalysis of minimally invasive versus open approach for pancreaticoduodenectomy. Surg Endosc 2016;30:5173-84.

11 Palanivelu C, Senthilnathan P, Sabnis SC, et al. Randomized clinical trial of laparoscopic versus open pancreatoduodenectomy for periampullary tumours. Br J Surg 2017;104:1443-50.

12 Poves I, Burdio F, Morato O, et al. Comparison of perioperative outcomes between laparoscopic and open approach for pancreatoduodenectomy: the PADULAP randomized controlled trial. Annals of surgery 2018;268:731-9.

13 van Hilst J, de Rooij T, Bosscha K, et al. Laparoscopic versus open pancreatoduodenectomy for pancreatic or periampullary tumours (LEOPARD-2): a multicentre, patient-blinded, randomised controlled phase 2/3 trial. The Lancet Gastroenterology \& Hepatology 2019;4:199-207.

14 Slankamenac K, Graf R, Barkun J, et al. The comprehensive complication index. A novel continuous scale to measure surgical morbidity. Ann Surg 2013;258:1-7.

15 Wang M, Peng B, Liu J, et al. Practice patterns and perioperative outcomes of laparoscopic pancreaticoduodenectomy in China: a retrospective multicenter analysis of 1029 patients. Annals of surgery 2019.
16 Asbun HJ, Conlon K, Fernandez-Cruz L, et al. When to perform a pancreatoduodenectomy in the absence of positive histology? A consensus statement by the International Study Group of Pancreatic Surgery. Surgery 2014;155:887-92.

17 Nccn clinical practice guidelines in oncology: pancreatic adenocarcinoma. V2., 2019. Available: https://www.nccn.org/ professionals/physician gls/pdf/pancreatic.pdf

18 Tol JAMG, Gouma DJ, Bassi C, et al. Definition of a standard lymphadenectomy in surgery for pancreatic ductal adenocarcinoma: a consensus statement by the International Study Group on pancreatic surgery (ISGPS). Surgery 2014;156:591-600.

19 Wang $\mathrm{M}$, Xu S, Zhang $\mathrm{H}$, et al. Imbedding pancreaticojejunostomy used in pure laparoscopic pancreaticoduodenectomy for nondilated pancreatic duct. Surg Endosc 2017;31:1986-92.

20 Wang S, Shi N, You L, et al. Minimally invasive surgical approach versus open procedure for pancreaticoduodenectomy. A systematic review and meta-analysis. Medicine 2018;96:e8619.

21 Chun YS, Pawlik TM, Vauthey J-N. 8Th edition of the AJCC cancer staging manual: pancreas and hepatobiliary cancers. Ann Surg Oncol 2018;25:845-7.

22 Bosman F, Carneiro F, Hruban R, et al. Who classification of tumours of the digestive system. 4th edition. IARC, 2010.

23 Clavien PA, Barkun J, de Oliveira ML, et al. The Clavien-Dindo classification of surgical complications: five-year experience. Ann Surg 2009;250:187-96.

24 Chan A-W, Tetzlaff JM, Altman DG, et al. Spirit 2013 statement: defining standard protocol items for clinical trials. Ann Intern Med 2013;158:200-7.

25 Moher D, Hopewell S, Schulz KF, et al. D, Egger M, Altman DG: CONSORT 2010 Explanation and Elaboration: Updated guidelines for reporting parallel group randomised trials. Journal of clinical epidemiology 2010, 63:e1-37. 